\section{ID-Infection Control Position. BC/BE}

\section{UNIVERSITY OF ROCHESTER SCHOOL OF MEDICINE}

University of Rochester School of Medicine is seeking a junior or mid-level faculty member to join a 3 person Infectious Disease unit at Rochester General Hospital, Rochester, NY. Opportunity to work as the hospital epidemiologist with four infection control practitioners at a 500 bed busy community hospital with nationally recognized innovative programs in infection control. Other duties include medical student and resident teaching and infectious disease consultations. Active federally funded research program is ongoing with ample opportunities for clinical and translational research.

Please send letter of interest and curriculum vitae to Edward E. Walsh, MD ( Edward.walsh@rochestergeneral.org ), Infectious Disease, University of Rochester, Rochester General Hospital, 1425 Portland Avenue, Rochester, NY.

\section{Classified Advertising Information}

\section{Classified Rates}

$1 \mathbf{x}-\$ 215$ for 50 words or less; $\$ 1.00$ for each word over 50

\section{Typesetting Services}

All classified ads are typeset in the same font and format. However, bold, italicized and underlined typefaces are available for $50 \phi$ extra per word.

\section{Word Count}

In calculating your cost, we define a word as one or more letters surrounded by spaces. Below are some examples:

$\begin{array}{ll}\text { Maria A. Johnson, MD } & 4 \text { words } \\ \text { October } 9,2003 & 3 \text { words } \\ 773-702-7600 & 1 \text { word } \\ \text { Chicago, IL } 60637 & 3 \text { words } \\ \text { a } & 1 \text { word } \\ \text { multi-specialty } & 1 \text { word }\end{array}$

To place a classified ad, or for more information, please contact:

DeLisa New

The University of Chicago Press

Phone: 773-702-5851

Fax: 773-753-3616

dnew@press.uchicago.edu

\title{
The difference between a career and
}

a purpose is about 8,000 miles.

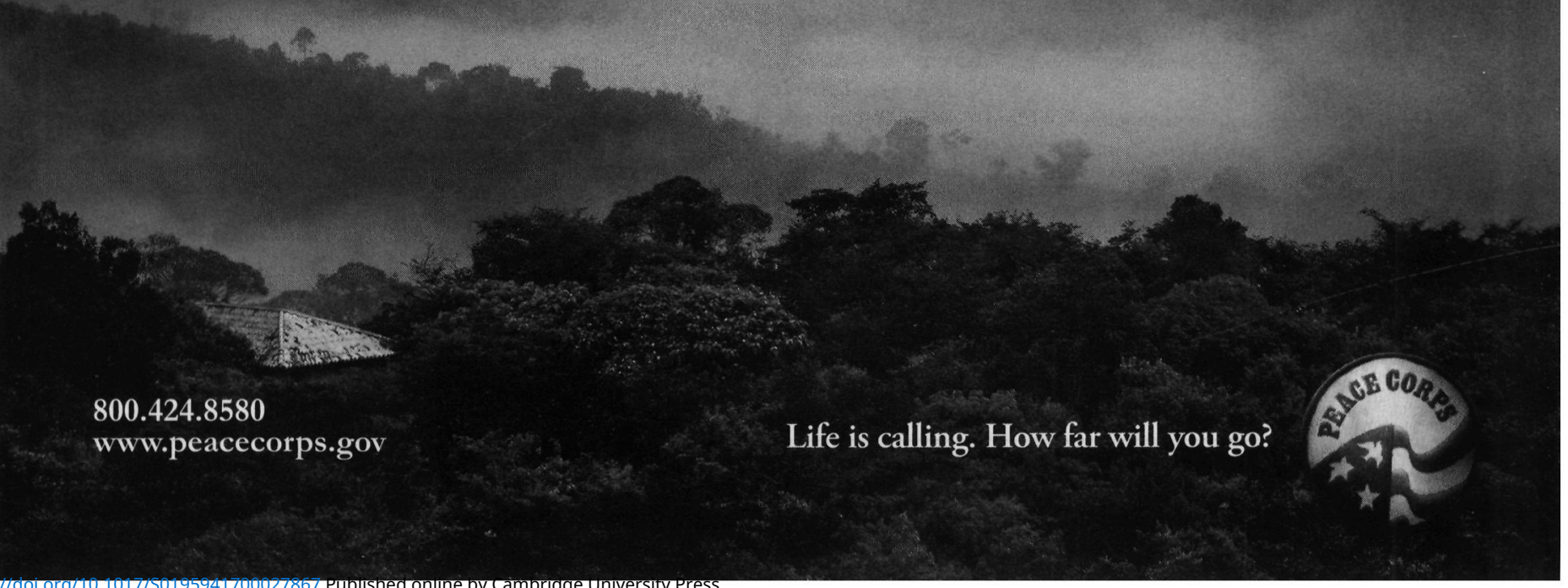




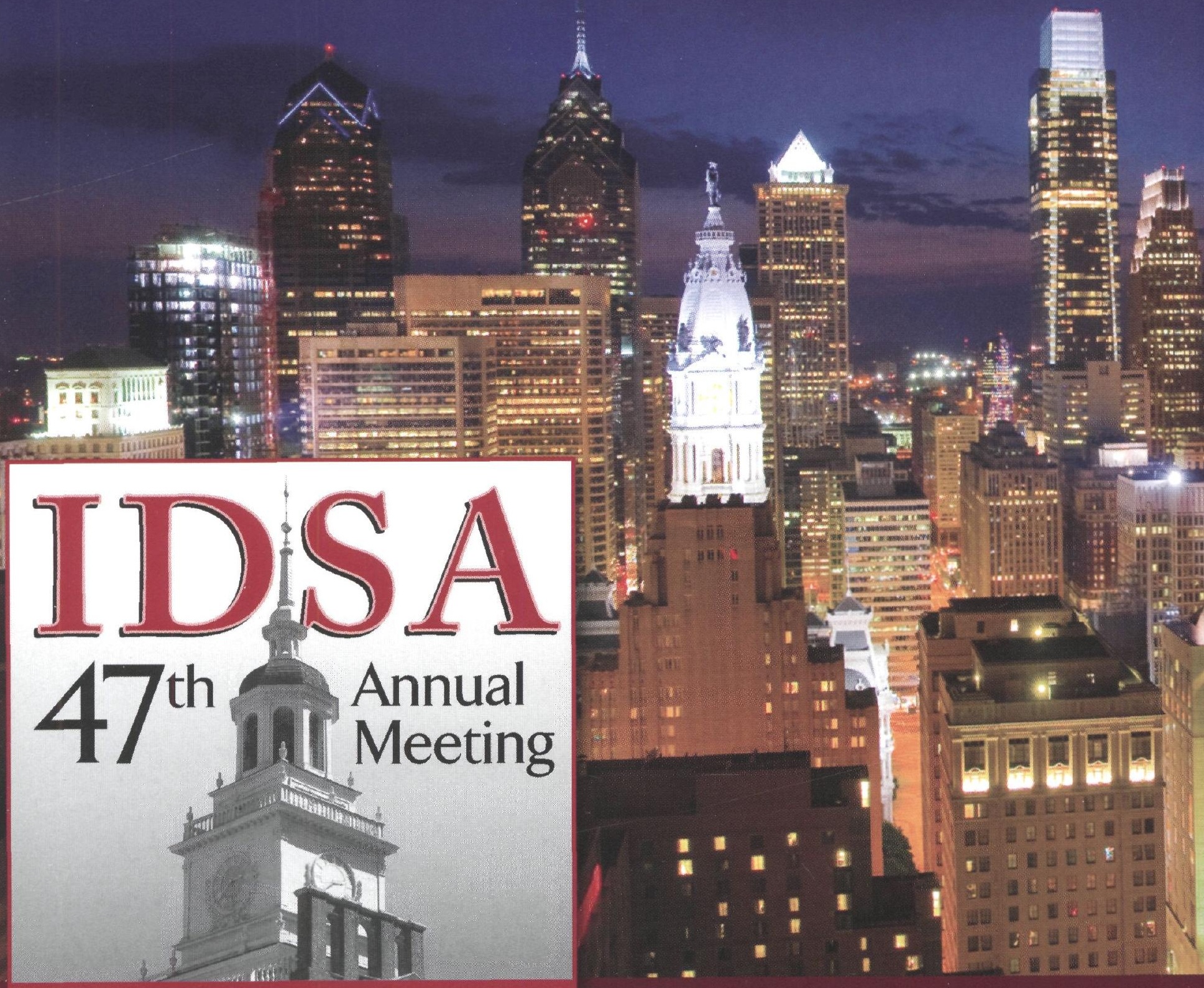

Oct. 29 - Nov. 1, 2009 Philadelphia, PA

For details on:

- Important Dates

- Preliminary Program

- Registration and Housing Online

Please visit www.idsociety.org/meetings

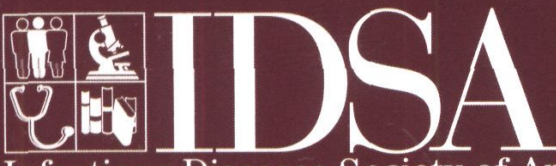

Infectious Diseases Society of America hivma

hiv medicine association 


\section{CHG}

protection

now comes
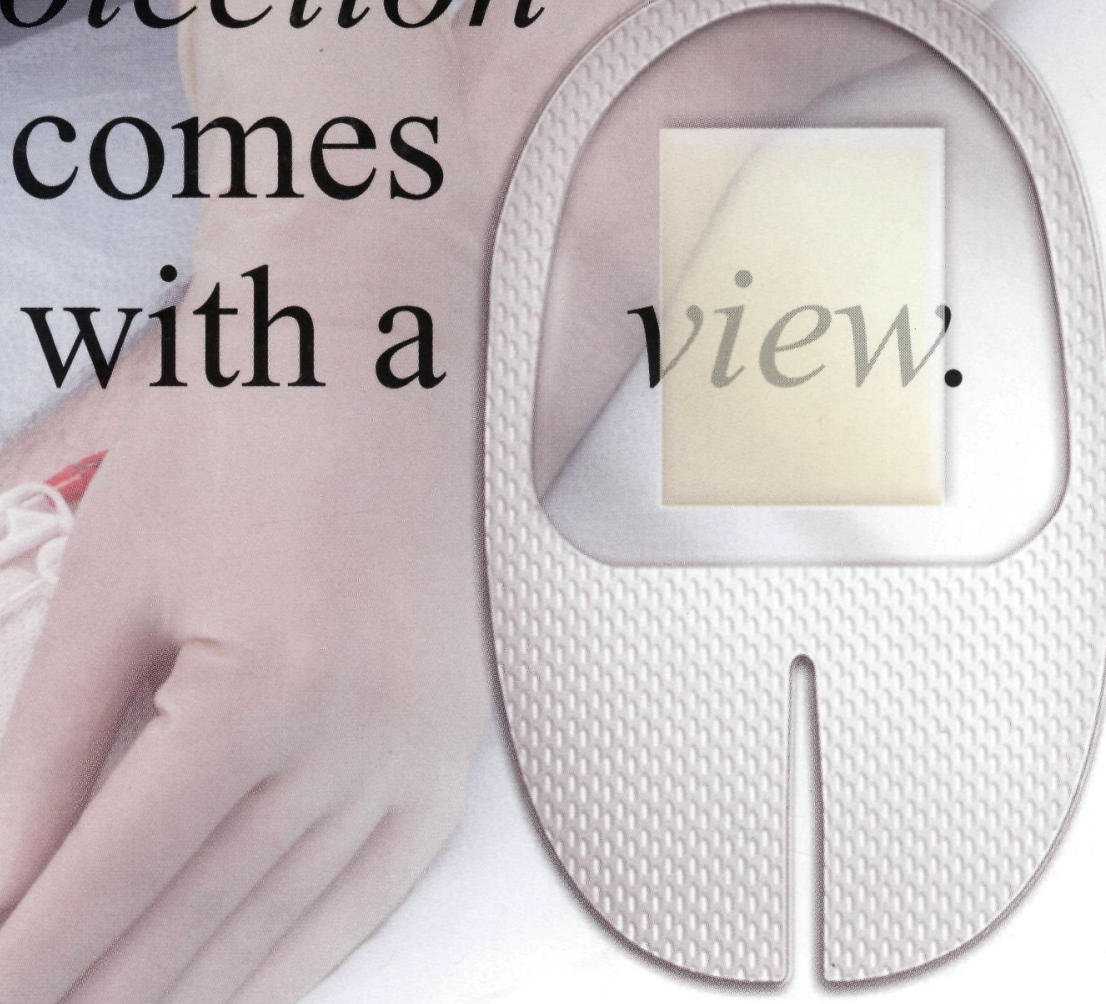

\section{Introducing $3 \mathrm{M}^{\mathrm{TM}}$ Tegaderm ${ }^{\mathrm{TM}} \mathrm{CHG}$}

\section{Chlorhexidine Gluconate IV Securement Dressing.}

If you're looking for a way to help staff comply with your protocols and deliver best patient care, the answer is finally clear. New Tegaderm ${ }^{\mathrm{TM}}$ CHG integrates antimicrobial protection with the transparent dressing that's easy to use. So you get the same protection every time.

- Clear, allowing continuous visualization of the insertion site

- Proven to be as effective as, or better at, reducing skin flora on healthy volunteers for up to 10 days than BIOPATCH ${ }^{\circledR}$ and more effective at preventing re-growth at 7 days

- Integrated design of dressing and $\mathrm{CHG}$ gel pad reduces application steps and minimizes potential for application error

\section{A new look at IV site protection.}

Visit www.3M.com/tegadermchg for more information. 